Im Blickpunkt

\section{Die Fachinformation}

Verständlich oder verwirrend für den Verordner?

Christine Greiner

\section{Literatur kompakt}

16 Probiotika zur Allergie-Primärprävention: schwache Evidenz

17 Radiochemotherapie oder Operation bei HNSCC?

18 CRS: Obstruktive Schlafapnoe mindert Erfolg der Sinuschirurgie

18 Oropharynx-Op und Septumplastik geht in einem Aufwasch

\section{Fortbildung}

\section{Ohrchirurgie}

Kein Operationserfolg ohne sorgfältige Nachsorge OlafMichel

24 Tubendilatation - ein neuartiges Therapieverfahren

Zehn Fragen, zehn Antworten

Eike Krause

28 CME: Orale Mukositis

Empfehlungen zu Prophylaxe und Therapie

Dorothea Riesenbeck, Annette Freidank, Johann Beck-Mannagetta, Anja Beylich

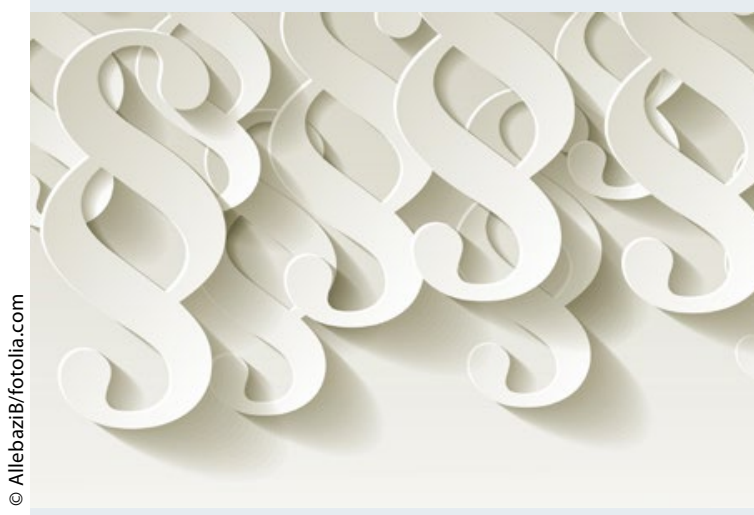

\section{Wichtige Informationen}

Während die Packungsbeilage eines Arzneimittels vor allem an den Patienten gerichtet ist, wendet sich die Fachinformation an die Fachkreise, also an Ärzte und Apotheker. Lesen Sie, was Verordner zum Inhalt der Fachinformation wissen müssen.

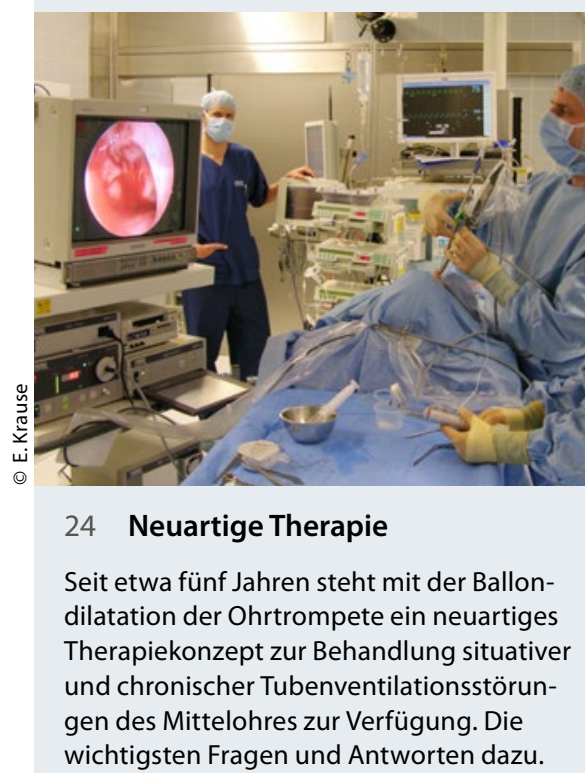

\begin{tabular}{lll}
\multicolumn{2}{l}{ Rubriken } & Sonderpublikation \\
3 & Editorial & Bitte beachten Sie die Sonderpublikation \\
8 & Panorama & "Akute Rhinosinusitis: Weniger Antibiotika - \\
52 & Industrieforum & mehr Phytotherapie" auf S. 56/57 in diesem \\
63 & Vorschau & \\
63 & Impressum &
\end{tabular}

Titel

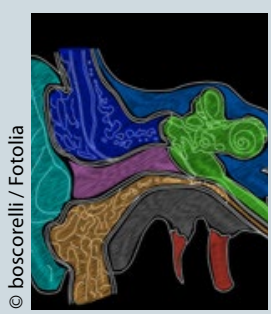

Sind Beschwerden auf eine ungenügende Öffnung der Ohrtrompete zurückzuführen, kann eine Ballondilatation helfen. 


\section{Hier steht eine Anzeige.}

Springer 


\section{Medizin aktuell}

\section{Kongressbericht 49. Fortbildungsveranstaltung für HNO-Ärzte HNO-Heilkunde interdisziplinär}

\section{Consilium HNO}

Sollten Taucher Ohrentropfen mit Ciprofloxacin vorbeugend anwenden?

\section{0 Ärztestudie}

Stimmt Ihre Work-Life-Balance? - Machen Sie den Test!

\section{Praxis konkret}

43 Galenus-von-Pergamon-Preis 2015

Ausgezeichnete Innovatoren

44 Verschreibungspflicht(en) von Arzneimitteln Bürokraten verordnen neue Rezeptformvorgaben

46 Der Kaufvertrag muss eigene Interessen decken Rechtliche Aspekte beim Praxiserwerb

48 Gewaltandrohung in der Praxis Klare Grenzen aufzeigen

49 „Es muss Spaß machen, bei Ihnen zu arbeiten!“ Personalführung als Baustein für den Praxiserfolg

50 Social Media in der Arztpraxis

Mit Facebook Image und Bekanntheit steigern

\section{Prisma}

\section{Jacques Joseph zum 150. Geburtstag}

Wegbereiter der plastischen Gesichts- und Nasenchirurgie W. Briedigkeit t, H. Behrbohm

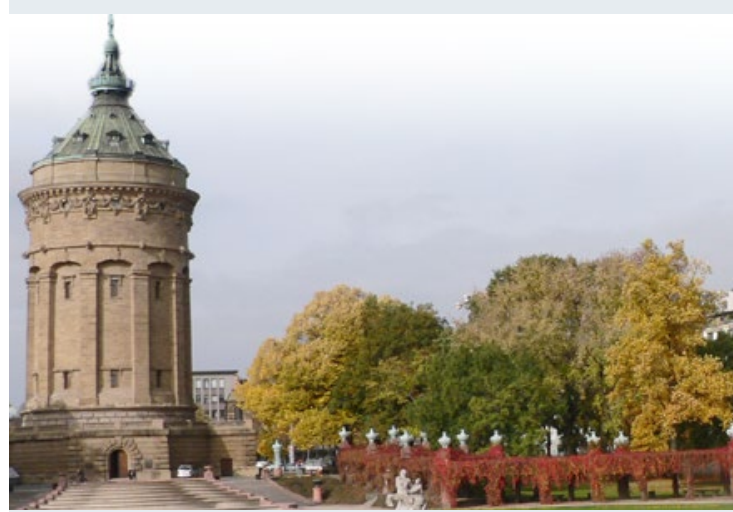

\section{Highlights aus Mannheim}

"Interdisziplinäre Aspekte in der HNOHeilkunde" war das Motto der diesjährigen Fortbildungsveranstaltung für HNO-Ärzte, in Mannheim. Das breite Themenspektrum der Vorträge zeigte die zentrale Stellung der HNO-Heilkunde innerhalb der medizinischen Fachdisziplinen.

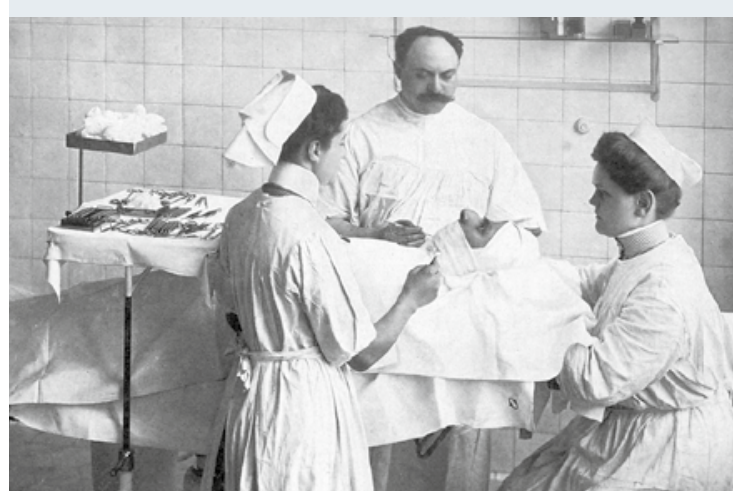

58 Wegbereiter der Nasenchirurgie

Nur spärliche Hinweise finden sich in der "Hall of Fame" der berühmten deutschen Chirurgen auf Jacques Joseph, einen Mann, dem heute bedeutende Verdienste für die plastische Gesichts- und besonders die Nasenchirurgie zugesprochen werden.

\section{Verlagsredaktion}

Gabriele Zörrgiebel

Springer Medizin Verlag GmbH

Aschauer Straße. 30

81549 München

E-Mail: gabriele.zoerrgiebel@springer.com

\section{Chefredaktion}

Prof. Dr. med. Gerhard Grevers

Nasen-Zentrum Starnberg

Prinzenweg 1

82319 Starnberg

\section{Mitmachen und gewinnen}

Es geht ganz einfach: Am Ende dieser Ausgabe (auf Seite 62) präsentieren wir Ihnen neun Titelbilder der HNO-Nachrichten. Wählen Sie daraus Ihren Favoriten, und mit ein bisschen Glück gewinnen Sie vielleicht sogar den ersten Preis: eine Canon Digital-Kamera. 